\title{
STELLAR AND IONIZED GAS-VELOCITY FIELDS IN THE CENTRAL REGIONS OF A SAMPLE OF GALAXIES
}

\author{
C. DEL BURGO, S. ARRIBAS, E. MEDIAVILLA AND B. GARCíA-LORENZO \\ Instituto de Astrofísica de Canarias \\ E-38200 La Laguna, Tenerife, Canary Islands, Spain
}

\begin{abstract}
We present the velocity fields for the ionized gas and the stars obtained from 2D fibre spectroscopy in the central regions of a sample of active (NGC 985, NGC 1052, NGC 6500) and non-active (M31, M32) galaxies.
\end{abstract}

\section{Observations and Results}

We are performing a long-term proyect with $2 \mathrm{D}$ fibre spectroscopy to study the kinematics of the ionized gas and the stars of the circumnuclear regions of galaxies (in this case NGC 1068: García-Lorenzo et al.). The 2D data allow us to discuss and compare the stellar and ionized gas velocity fields in galactic nuclei, overcoming the geometrical ambiguities common to $1 \mathrm{D}$ studies (long-slit, etc.).

The present data were obtained (with a seeing of $\sim 1^{\prime \prime}$ ) in September 1994 using the optical fibre system 2D-FIS (García et al.), which links the Cassegrain focus of the $4.2 \mathrm{~m}$ WHT with the ISIS double spectrograph. In the blue and red arms of ISIS, the spectral resolutions were of $1.5 \AA$ and 5 $\AA$, respectively. The $2 \mathrm{D}$-FIS data were reduced (using the IRAF package) basically in the same way as long-slit spectroscopy.

Here we present preliminary results: In NGC 1052 the stars seem to rotate while the ionized gas seems to be affected by radial motions. In the case of NGC 6500 the evidence is less compelling, but the differences suggest a decoupling of the stars from the ionized gas. In M31 we observe that the velocity dispersion centre is shifted towards the SW with respect to the stellar kinematic centre.

We thank A. García his help with 2D-FIS and all the staff of the ING/ORM for their kind support. This work has been partially supported 

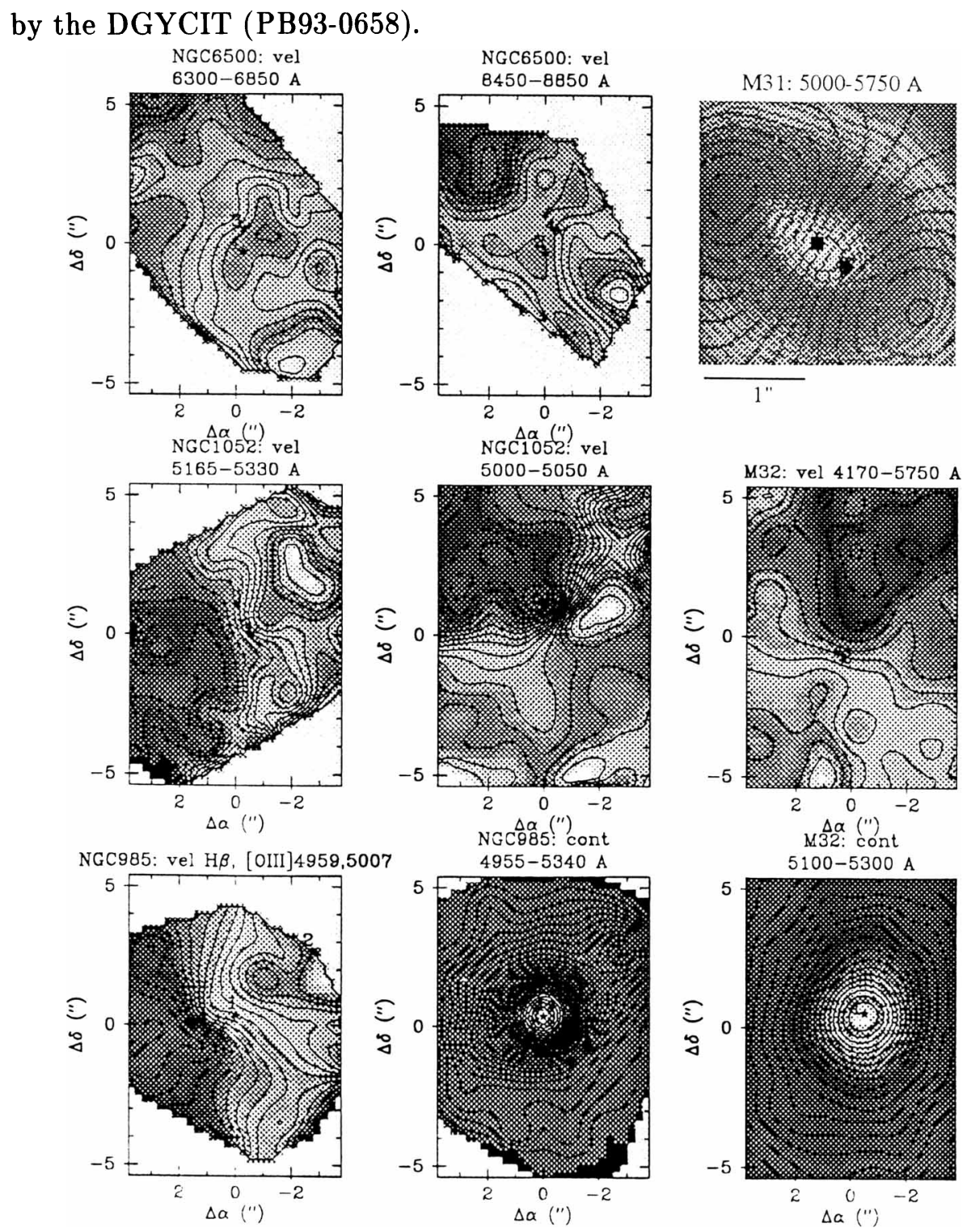

Figure 1. Top: NGC6500: stellar velocity field (SVF) and ionized gas velocity field (IGVF); M31: SVF overlayed on intensity map (IM). Middle: NGC 1052: SVF and IGVF; M32: SVF. Bottom: NGC 985: IGVF and IM; M32: IM.

\section{References}

García, A., Rasilla, J. L., Arribas, S., and Mediavilla, E. (1994): Instrumentation in Astronomy, Vol 2198, ed. D. L. Crawford and C. R. Craine (Hawaii: SPIE), 75

García-Lorenzo B., Mediavilla E., Arribas S. \& del Burgo C. (1997): ApJL, 483, 99 